\title{
The Impact of Noninvasive Prenatal Testing on the Practice of Maternal-Fetal Medicine
}

\author{
Lara A. Friel, MD, $\mathrm{PhD}^{1}$ Jennifer L. Czerwinski, $\mathrm{MS}^{1}$ \\ ${ }^{1}$ Department of Obstetrics, Gynecology, and Reproductive Sciences, \\ The University of Texas Health Science Center at Houston, Houston, \\ Texas \\ 2 Department of Obstetrics, Gynecology, and Reproductive Sciences \\ and Pediatrics, The University of Health Science Center at Houston, \\ Houston, Texas
}

\author{
Claire N. Singletary, MS²
}

\begin{abstract}
Address for correspondence Lara A. Friel, MD, PhD, Division of Maternal-Fetal Medicine, Department of Obstetrics, Gynecology, and Reproductive Sciences, UT Health - Houston, 6431 Fannin, MSB 3.286, Houston, TX 77030 (e-mail: Lara.a.friel@uth.tmc.edu).
\end{abstract}

\begin{abstract}
Keywords

- noninvasive prenatal testing

- NIPT

- cell-free DNA

- genetic screening

Objective Noninvasive prenatal testing (NIPT) via cell-free fetal DNA in the maternal circulation is a highly sensitive and specific new testing option. The objective of this study was to determine the impact of NIPT on the uptake of first trimester screening (FTS) and invasive genetic testing.

Study Design Uptake of prenatal testing was investigated in women referred for advanced maternal age or abnormal screening to the University of Texas Health Maternal-Fetal Medicine Clinics in Houston. Patients who presented from August to November 2011, before clinical introduction of NIPT, were compared with patients who presented from March to June 2012, after its introduction.

Results In patients referred between 14 and 22 weeks gestational age, invasive genetic testing was significantly reduced following the introduction of NIPT (35.4 vs. $17.9 \%, p<0.05)$. For patients referred at $<14$ weeks gestational age, FTS was significantly reduced with NIPT introduction (89.1 vs. 59.1\%, $p<0.05$ ); however, invasive genetic testing was not significantly different ( 20.0 vs. $14.0 \%, p>0.05$ ).

Conclusion NIPT has made an impact on the practice of maternal-fetal medicine by significantly decreasing the number of second trimester diagnostic tests performed. In addition, patients interested in early screening information appear to prefer the higher sensitivity and specificity of NIPT.
\end{abstract}

Identifying pregnancies at increased risk for aneuploidy has been a part of the practice of maternal-fetal medicine for several decades. Originally, women were identified based on maternal age alone and given the option of diagnostic testing via chorionic villus sampling (CVS) or amniocentesis to determine the fetal karyotype. Recently, maternal serum screening tests, with or without ultrasound findings, were developed for use in the general obstetrical population to identify at-risk pregnancies. ${ }^{1,2}$ Since CVS and amniocentesis carry a risk for miscarriage, research has continued to pursue other means of obtaining the fetal genetic complement.

received

May 30, 2013

accepted after revision

September 19, 2013

published online

December 11, 2013
Recently, three separate multicenter trials of noninvasive prenatal testing (NIPT) showed that analyzing cell-free DNA in the maternal circulation results in a highly sensitive and specific testing option for fetal aneuploidy, with 98 to $100 \%$ detection rate at $<0.3 \%$ false-positive rate for Down syndrome and 97 to $100 \%$ detection rate at $<0.28 \%$ false-positive rate for trisomy $18 .^{3-6}$ The detection rate for trisomy 13 varied more widely across studies, ranging from 79 to $92 \%$ with $<1 \%$ false-positive rate. ${ }^{4,6}$

Advances in prenatal screening for aneuploidy have led to a decrease in invasive testing and an increase in screening in the
Copyright $\odot 2014$ by Thieme Medical Publishers, Inc., 333 Seventh Avenue, New York, NY 10001, USA. Tel: +1(212) 584-4662.
DOI http://dx.doi.org/ $10.1055 / \mathrm{s}-0033-1359717$. ISSN 0735-1631. 
at-risk population. ${ }^{7,8}$ Even women who elect amniocentesis report misgivings about placing their pregnancy at risk. ${ }^{9}$ The high detection rate and low false-positive rate for fetal aneuploidy demonstrated by NIPT coupled with the lack of risk to the fetus poises this new technology to have significant impact on invasive testing. We conducted a retrospective study to compare testing uptake before the introduction of NIPT with testing uptake following its introduction to determine if NIPT significantly altered clinical practice. This information is important to demonstrate how new technologies impact patient decisions and the practice of maternal-fetal medicine.

\section{Materials and Methods}

A retrospective review of the prenatal genetic counseling database, which contains information from all patients having genetic counseling at the University of Texas Health Maternal-Fetal Medicine Clinics in Houston, was performed. The 4 months before the availability of NIPT (August 1, 2011, through November 30, 2011) and the 4 months after an established clinical NIPT protocol (March 1, 2012, through June 30, 2012) were compared to determine whether the uptake rates for invasive diagnostic testing had changed. The date of genetic counseling, indication, gestational age, number of gestations, gravidity, parity, and elected testing were obtained. Only singleton gestations having genetic counseling for advanced maternal age or positive maternal serum screening, with or without additional indications, before
22 weeks gestation were identified for statistical analysis. Patients pregnant with multiple gestations and those presenting after 22 weeks were not included, as the risks and accuracy of available testing options are different in these groups. Patients within each study time period were further divided into those seen before 14 weeks gestational age and those seen between 14 and 22 weeks, as unique screening and diagnostic testing options were available to each group. Finally, per capita prenatal testing reimbursement was calculated for each time period to determine whether the introduction of NIPT influenced clinical practice as measured by reimbursement. A model was created to study reimbursement in each group based on the distribution of patients within the two groups that were privately insured, Medicaid insured, or self-pay for the following procedures (first trimester ultrasound-76801, first trimester screen [FTS]-76813, CVS with ultrasound guidance-59015/76945, and amniocentesis with ultrasound guidance-59000/76946). Reimbursement for NIPT or other laboratory charges were not included in the analysis, as these are not captured by our Maternal-Fetal Medicine clinics. For the patients with private insurance, procedure reimbursement was calculated based on the average of the rates of our two largest private insurers (Blue Cross Blue Shield and United Health Care). The collection of data for research was approved by the Institutional Review Board of the University of Texas Health Science Center at Houston IRB \#HSC-MS-12-0384. The statistical package employed was SPSS 20 (SPSS Inc, Chicago, IL). Comparison of proportions

Table 1 Demographic and clinical characteristics of the study groups

\begin{tabular}{|c|c|c|c|c|}
\hline Clinical characteristics & $\begin{array}{l}\text { Pre-NIPT } \\
<14 \text { wk GA } \\
(n=165)\end{array}$ & $\begin{array}{l}\text { Post-NIPT } \\
<14 \text { wk GA } \\
(n=193)\end{array}$ & $\begin{array}{l}\text { Pre-NIPT } \\
14-22 \text { wk GA } \\
(n=294)\end{array}$ & $\begin{array}{l}\text { Post-NIPT } \\
14-22 \text { wk GA } \\
(n=330)\end{array}$ \\
\hline Maternal age (y) & $\begin{array}{l}37.2 \\
(26.1-43.9)\end{array}$ & $\begin{array}{l}37.2 \\
(23.9-46.2)\end{array}$ & $\begin{array}{l}35.7 \\
(16.7-44.5)\end{array}$ & $\begin{array}{l}36.7 \\
(18.9-46.6)\end{array}$ \\
\hline Gravidity & $\begin{array}{l}3 \\
(1-10)\end{array}$ & $\begin{array}{l}3 \\
(1-11)\end{array}$ & $\begin{array}{l}3 \\
(1-9)\end{array}$ & $\begin{array}{l}3 \\
(1-12)\end{array}$ \\
\hline Parity & $\begin{array}{l}1 \\
(0-7)\end{array}$ & $\begin{array}{l}1 \\
(0-6)\end{array}$ & $\begin{array}{l}1 \\
(0-7)\end{array}$ & $\begin{array}{l}1 \\
(0-10)\end{array}$ \\
\hline \multicolumn{5}{|l|}{ Race (\%) } \\
\hline African American & $13.3(22)$ & $14.5(28)$ & $17.0(50)$ & $18.2(60)$ \\
\hline Asian & $24.2(40)$ & $20.2(39)$ & $15.6(46)$ & $19.4(64)$ \\
\hline Caucasian & $36.4(60)$ & $32.6(63)$ & $23.8(70)$ & $15.8(52)$ \\
\hline Hispanic & $24.2(40)$ & $28.0(54)$ & $41.2(121)$ & $43.9(145)$ \\
\hline Other & $1.8(3)$ & $2.1(4)$ & $2.4(7)$ & $2.1(7)$ \\
\hline \multicolumn{5}{|l|}{ Insurance (\%) } \\
\hline Medicaid & $9.1(15)$ & $14.5(28)$ & $45.9(135)$ & $46.4(153)$ \\
\hline Private & $85.5(141)$ & $79.3(153)$ & $52.4(154)$ & 48.2 (159) \\
\hline Other & $2.4(4)$ & $2.6(5)$ & $0.7(2)$ & $2.4(8)$ \\
\hline GA at the time of genetic counseling (wk) & $12.1(8.7-13.9)$ & $12.2(10.0-13.9)$ & $18.3(14.4-21.9)$ & $18.9(14.0-21.9)$ \\
\hline
\end{tabular}

Abbreviations: GA, gestational age; NIPT, noninvasive prenatal testing; wk, weeks, y, years.

Notes: Values are expressed as percentage (number) or median (range). Data regarding gravidity and parity were missing for 85 patients. Data regarding race were missing for seven patients. Data regarding insurance were missing for 25 patients. 
was performed by Pearson chi-square test. A $p$ value of $<0.05$ was considered statistically significant.

\section{Results}

A total of 792 patients received genetic counseling between August 1, 2011 and November 30, 2011. Of those, 459 (58\%) were seen before 22 weeks for advanced maternal age and/or abnormal genetic screen. Of the 838 patients seen between March 1, 2012 and June 30, 2012, 523 (62.4\%) were seen before 22 weeks for advanced maternal age and/or abnormal genetic screen. Maternal age, gravidity, parity, race, insurance, and gestational age at the time of genetic counseling was not significantly different $(p>0.05)$ in the pre- and postNIPT groups when compared with the same gestational age subgroup (-Table $\mathbf{1}$ ).

Overall, NIPT was chosen by $31.6 \%$ of patients who received genetic counseling in the first trimester and by $17.0 \%$ in the second trimester. Before the implementation of NIPT, 89.1\% $(n=147)$ of patients referred before 14 weeks elected to pursue combined FTS. The uptake of FTS fell significantly to $59.1 \%(n=114)$ following the introduction of NIPT $(p<0.05$; - Fig. 1). The uptake of invasive genetic testing by first trimester patients was not significantly different with $20.0 \%(n=33)$ pursuing CVS or amniocentesis before NIPT and a $14.0 \%(n=27)$ uptake following the availability of NIPT $(p>0.05$; - Fig. 2). There was, however, a significant decrease in the uptake of invasive diagnostic testing for those patients receiving genetic counseling between 14 and 22 weeks with $35.4 \%(n=104)$ of patients before NIPT electing amniocentesis and only $17.9 \%(n=59)$ of patients electing amniocentesis after the availability of NIPT $(p<0.05 ;$ - Fig. 3). Although there was a decrease in the uptake of amniocentesis and an increase in uptake of NIPT, overall, there was no difference in the number of women choosing no further genetic testing following genetic counseling after the introduction of NIPT compared with before its introduction ( 42.4 vs. $41.4 \% ; p<0.05$ ). Finally, per capita testing reimbursement was $15.77 \%$ less following the introduction of NIPT (\$101.32 vs. \$85.34).

\section{Comment}

\section{Principal Findings}

NIPT has already made an impact on our practice of maternal-fetal medicine by significantly decreasing the number of second trimester diagnostic tests performed. This finding was also observed by another group when only women with a positive aneuploidy screening result were studied. ${ }^{10}$ Patients interested in early screening information appear to prefer the higher sensitivity and specificity of NIPT to combined FTS. Early diagnostic information seekers, however, appear to remain most comfortable with CVS.

Per capita testing reimbursement fell by almost $16 \%$. It is important to note that it is our practice to perform an ultrasound for viability at the time of NIPT testing to ensure that NIPT is indicated. Per capita testing reimbursement could fall even more sharply in units that have not adopted this practice. For example, if our practice did not include a viability ultrasound before blood draw for NIPT in the first trimester, a per capita drop of $35.37 \%$ would have occurred ( $\$ 101.32$ vs. $\$ 65.48$ ). Maternal-Fetal Medicine clinics will need to take into consideration decreased uptake of second trimester diagnostic tests and the utilization of viability ultrasounds when evaluating their business model.

In contrast with the recent findings of Chetty et $\mathrm{al}^{10}{ }^{10}$ our study did not identify a decline in women choosing no further genetic testing following the introduction of NIPT. This may be due to the many differences in the patient populations studied. Our patients were identified by referrals for genetic counseling for both advanced maternal age and positive

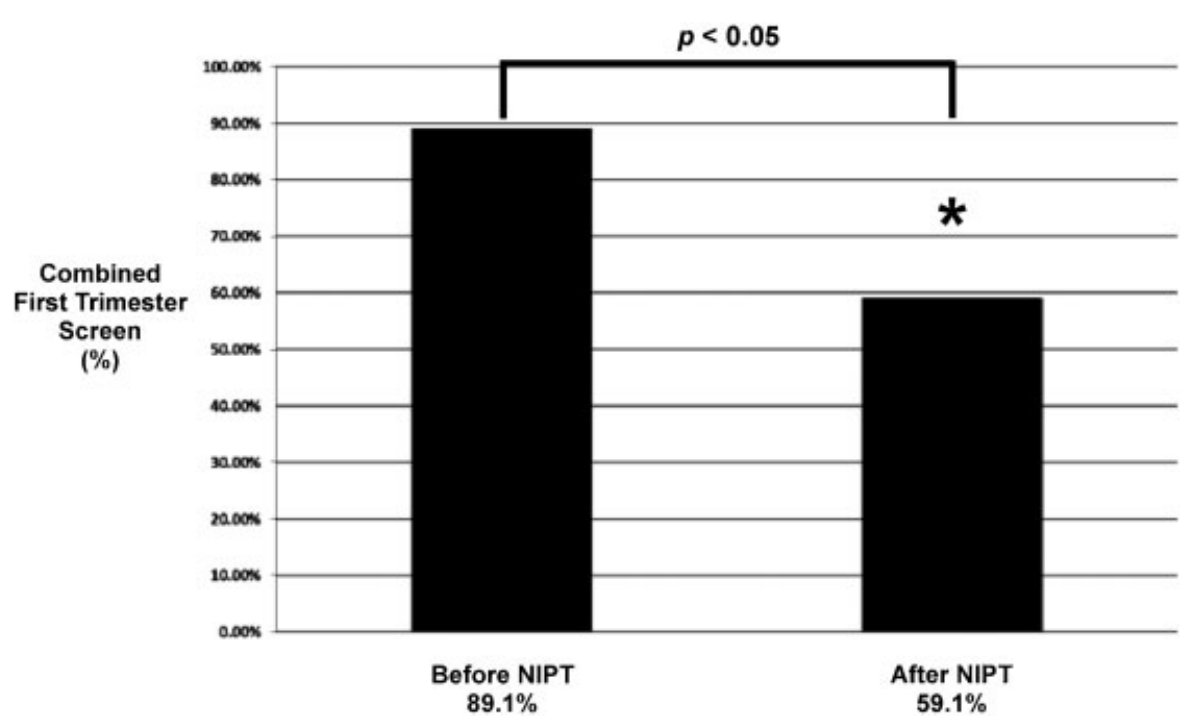

Fig. 1 The uptake of FTS for patients receiving genetic counseling at $<14$ weeks gestational age before and after the availability of NIPT. Before the implementation of NIPT, $89.1 \%(n=147)$ of patients referred at $<14$ weeks elected to pursue FTS. This uptake fell significantly to $59.1 \%(n=114)$ following the introduction of NIPT $\left(p<0.05 ;{ }^{*}\right)$. FTS, first trimester screening; NIPT, noninvasive prenatal testing. 


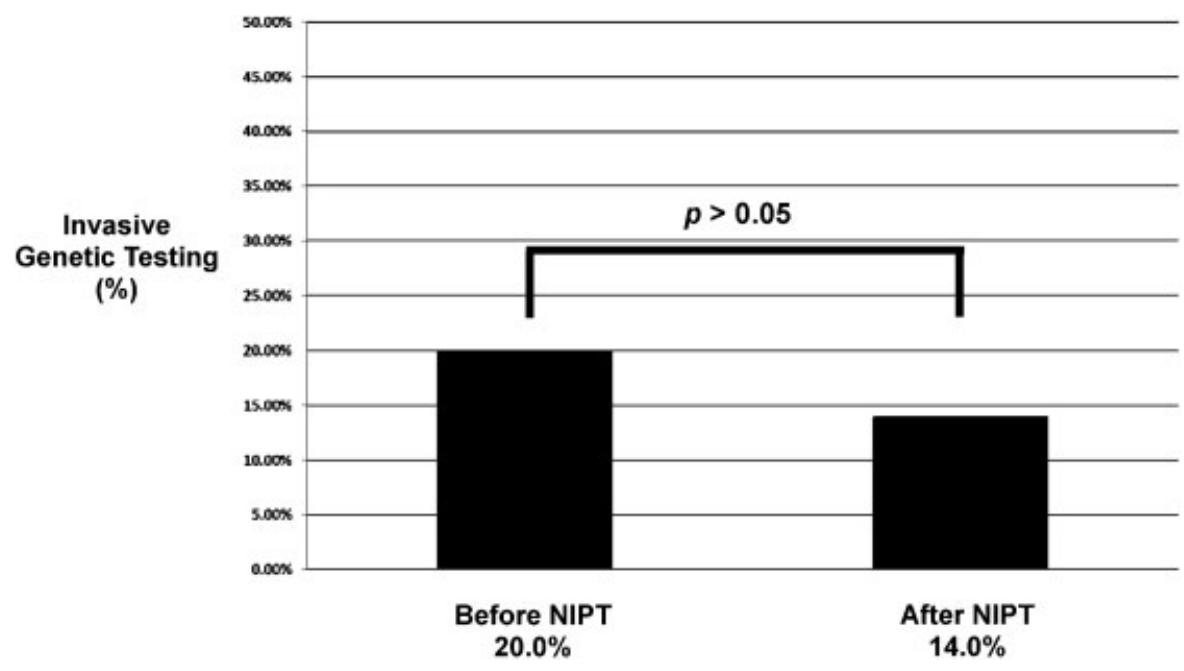

Fig. 2 The uptake of invasive diagnostic testing for patients receiving genetic counseling at $<14$ weeks gestational age before and after the availability of NIPT. The uptake of invasive genetic testing by first trimester patients was not significantly different with $20.0 \%(n=33)$ pursuing CVS or amniocentesis before NIPT and a $14.0 \%(n=27)$ uptake following the availability of NIPT $(p>0.05)$. CVS, chorionic villus sampling; NIPT, noninvasive prenatal testing.

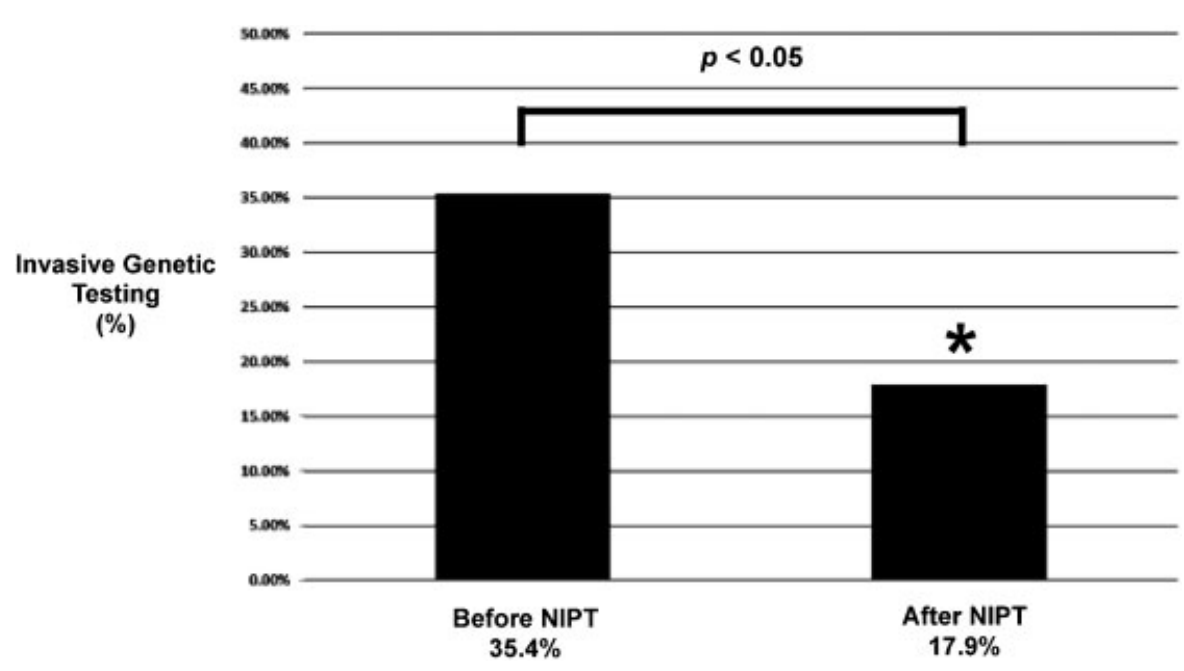

Fig. 3 The uptake of invasive diagnostic testing for patients receiving genetic counseling between 14 and 22 weeks before and after the availability of NIPT. There was a significant decrease in the uptake of invasive diagnostic testing for those patients receiving genetic counseling between 14 and 22 weeks with 35.4\% ( $n=104)$ of patients before NIPT electing amniocentesis and only $17.9 \%(n=59)$ of patients electing amniocentesis after the availability of NIPT $\left(p<0.05 ;{ }^{*}\right)$. NIPT, noninvasive prenatal testing.

maternal serum screening in parallel to recent practice guidelines developed jointly by the American College of Obstetricians and Gynecologists Committee on Genetics and the Society for Maternal-Fetal Medicine Publications Committee for NIPT. ${ }^{11}$ Consequently, our patient population was older. Furthermore, they also had a different ethnic makeup and payer mix.

\section{Strengths and Weaknesses}

The strengths of our study include the evaluation of the immediate impact of this new genetic screening test in a large number of ethnically diverse patients from a single urban referral area with a uniform protocol for offering NIPT. The protocol was in accordance with recently published practice guidelines. ${ }^{11,12}$ Weaknesses include the fact that the study only evaluates the immediate impact of the intro- duction of NIPT. These data would be expected to change with increased knowledge and education about NIPT by both patients and referring physicians. Finally, there are regional differences in uptake of prenatal genetic screening and invasive diagnostic testing. Our results may be different than those found in other regions, as well as more rural areas, of the country.

\section{Implications for Clinicians}

It is imperative that clinicians educate themselves about NIPT, as its introduction is transforming prenatal testing. Taking a family history and reviewing patient records to determine that a patient is an appropriate candidate, and pretest counseling to ensure that patients understand the implication of a positive and negative result is essential. $^{12}$ 


\section{Unanswered Questions/Future Research}

Our study was not designed to look at how the test performs in our clinical setting, including investigation of false-positive and false-negative rates. Future studies will be needed to evaluate test performance outside of a clinical trial. It will also be important to investigate both patient and provider knowledge and understanding of NIPT.

\section{Conclusions}

NIPT has made an impact on the practice of maternal-fetal medicine by significantly decreasing the number of second trimester diagnostic tests performed and thus per capita testing reimbursement.

Notes

This study was performed in Houston, Texas, United States. The paper was presented at the 33rd Annual Meeting of the Society for Maternal-Fetal Medicine, San Francisco, California, February 11-16, 2013.

Conflict of Interest

The authors report no conflict of interest.

\section{References}

1 American College of Obstetricians and Gynecologists. ACOG Practice Bulletin No. 88, December 2007. Invasive prenatal testing for aneuploidy. Obstet Gynecol 2007;110(6):1459-1467

2 ACOG Committee on Practice Bulletins. ACOG Practice Bulletin No. 77: screening for fetal chromosomal abnormalities. Obstet Gynecol 2007;109(1):217-227
3 Palomaki GE, Kloza EM, Lambert-Messerlian GM, et al. DNA sequencing of maternal plasma to detect Down syndrome: an international clinical validation study. Genet Med 2011;13(11): 913-920

4 Bianchi DW, Platt LD, Goldberg JD, Abuhamad AZ, Sehnert AJ, Rava RP; MatErnal BLood IS Source to Accurately diagnose fetal aneuploidy (MELISSA) Study Group. Genome-wide fetal aneuploidy detection by maternal plasma DNA sequencing. Obstet Gynecol 2012;119(5):890-901

5 Norton ME, Brar H, Weiss J, et al. Non-Invasive Chromosomal Evaluation (NICE) Study: results of a multicenter prospective cohort study for detection of fetal trisomy 21 and trisomy 18 . Am J Obstet Gynecol 2012;207(2):e1-e8

6 Palomaki GE, Deciu C, Kloza EM, et al. DNA sequencing of maternal plasma reliably identifies trisomy 18 and trisomy 13 as well as Down syndrome: an international collaborative study. Genet Med 2012;14(3):296-305

7 Benn PA, Egan JF, Fang M, Smith-Bindman R. Changes in the utilization of prenatal diagnosis. Obstet Gynecol 2004;103(6): $1255-1260$

8 Nakata N, Wang Y, Bhatt S. Trends in prenatal screening and diagnostic testing among women referred for advanced maternal age. Prenat Diagn 2010;30(3):198-206

9 Sapp JC, Hull SC, Duffer S, et al. Ambivalence toward undergoing invasive prenatal testing: an exploration of its origins. Prenat Diagn 2010;30(1):77-82

10 Chetty S, Garabedian MJ, Norton ME. Uptake of noninvasive prenatal testing (NIPT) in women following positive aneuploidy screening. Prenat Diagn 2013;33(6):542-546

11 American College of Obstetricians and Gynecologists Committee on Genetics. Committee Opinion No. 545: Noninvasive prenatal testing for fetal aneuploidy. Obstet Gynecol 2012;120(6): 1532-1534

12 Devers PL, Cronister A, Ormond KE, Facio F, Brasington CK, Flodman P. Noninvasive prenatal testing/noninvasive prenatal diagnosis: the position of the National Society of Genetic Counselors. J Genet Couns 2013;22(3):291-295 